\section{Gènes anciens et gènes nouveaux dans la boîte à outils moléculaire de la cellule souche ancestrale des animaux}

Alexandre Alié ${ }^{1}$, Michaël Manuel ${ }^{2}$, Noriko Funayama ${ }^{3}$
1 Sorbonne Universités, UPMC Univ Paris 06, CNRS, laboratoire de biologie du développement de Villefranche-sur-mer (LBDV), station zoologique, 181, chemin du Lazaret, 06230 Villefranche-sur-Mer, France ; 2 Sorbonne Université, UPMC Univ Paris 06, CNRS, Département Évolution Paris Seine, Institut de Biologie Paris Seine, 75005 Paris, France;

${ }^{3}$ Department of Biophysics, Graduate School of Science, Kyoto University, Kyoto 606-8502, Japon. alexandre.alie@obs-vlfr.fr
> Les cellules souches sont des cellules le plus souvent indifférenciées ayant la capacité de produire d'autres types cellulaires tout en s'auto-renouvelant. Elles sont présentes chez tous les animaux et jouent un rôle central non seulement dans l'embryogenèse, mais également dans la production des gamètes, l'homéostasie des tissus, la régénération ou les nombreux modes de reproduction asexuée que l'on observe à travers l'arbre phylogénétique des métazoaires (comme le bourgeonnement, la fission, etc.) [1]. Par définition, les cellules souches n'existent pas chez les organismes unicellulaires, ce qui implique que leur acquisition est intimement liée à l'apparition de la multicellularité chez un ancêtre des métazoaires. Dès lors, comprendre les bases génétiques de l'acquisition des cellules souches peut nous aider à comprendre celles de l'émergence et de l'évolution des animaux.

Les éponges forment le groupe frère de tous les autres métazoaires (appelés eumétazoaires) [2, 3] et occupent à ce titre une position clé pour comprendre les premières étapes évolutives des animaux [15] $(\rightarrow)$.

Depuis 2010 , le

$\rightarrow$ Voir la Perspective de D. Aubert, $m / s n^{\circ} 5$, séquençage du mai 2016, page 497 génome de trois espèces d'éponges et des transcriptomes d'une dizaine d'autres a révélé la présence de la plupart des familles et sous-familles de gènes de développement connus chez l'homme ou la drosophile [4]. Ces approches ont également révélé certaines homologies entre le plan d'organisation des éponges et celui des eumétazoaires. Dans notre étude [5], nous nous sommes intéressés à l'origine des cellules souches en cherchant à reconstruire le répertoire de gènes ancestralement exprimés par ces cellules chez les animaux. Pour cela nous avons identifié des gènes homologues exprimés par les cellules souches d'éponges et celles d'autres lignées, afin de déterminer les innovations génétiques ayant accompagné l'acquisition de ce type cellulaire il y a 700 millions d'années.

Ephydatia fluviatilis est une éponge d'eau douce appartenant au groupe des démosponges. Elle se reproduit principalement de façon asexuée, lorsque l'éponge-mère produit des milliers de gemmules (des sphères mesurant un demi-millimètre de diamètre, enveloppées d'un squelette siliceux) contenant des centaines de cellules en dormance [6]. Lorsqu'une gemmule se sépare de l'éponge-mère et trouve un substrat approprié, les cellules en dormance migrent en dehors de la sphère et prolifèrent activement. On les appelle alors archéocytes. Ce sont des cellules souches totipotentes qui s'auto-renouvellent tout en produisant les différents types cellulaires contribuant à former une éponge juvénile fonctionnelle. Les archéocytes peuvent également former la lignée germinale femelle (les ovocytes) et entrent donc dans la catégorie de ce que l'on appelle les unlimited primordial stem cells (uPriSc) [7]. Les spermatozoïdes, quant à eux, proviennent de la transdifférenciation d'un autre type cellulaire, les choanocytes. Ceux-ci sont munis d'un flagelle entouré d'une collerette de microvillosités ayant pour rôle de créer un courant d'eau et de capturer les particules alimentaires. Les éponges contiennent également divers types cellulaires incluant, par exemple, plusieurs types de cellules épithéliales, des cellules formant le squelette siliceux, des cellules contrôlant le flux d'entrée d'eau ou encore des cellules immunitaires [6]. Durant la première étape de notre étude, nous avons obtenu une fraction pure d'archéocytes. Ceci a été rendu possible grâce à un marquage différentiel des différents types de cellules souches. Les archéocytes peuvent en effet être spécifiquement rendus fluorescents lorsque les éponges sont incubées en présence d'un marqueur mitochondrial, la rhodamine 123 (Rhol23), qui marque les plaquettes vitellines des archéocytes. Les choanocytes peuvent, eux, être spécifiquement marqués en nourrissant les éponges avec des microbilles fluorescentes qui sont ingérées comme s'il s'agissait de particules alimentaires. Les autres types cellulaires ne sont pas marqués par ces deux traitements. En utilisant le tri cellulaire par FACS ${ }^{1}$ (fluorescence-activated cell sorting), nous avons donc pu isoler trois fractions cellulaires à partir d'éponges marquées: des archéocytes à partir d'éponges marquées à la Rhol23, des choanocytes à partir d'éponges

Type de cytométrie de flux basé sur la fluorescence des cellules permettant leur tri. 
A Germination de la gemmule et migration des archéocytes

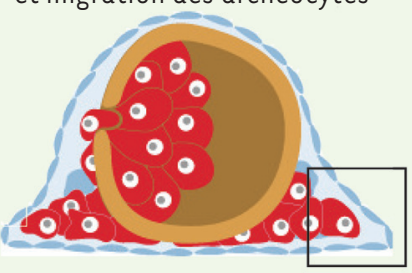

Archéocytes marqués (Rhol23)

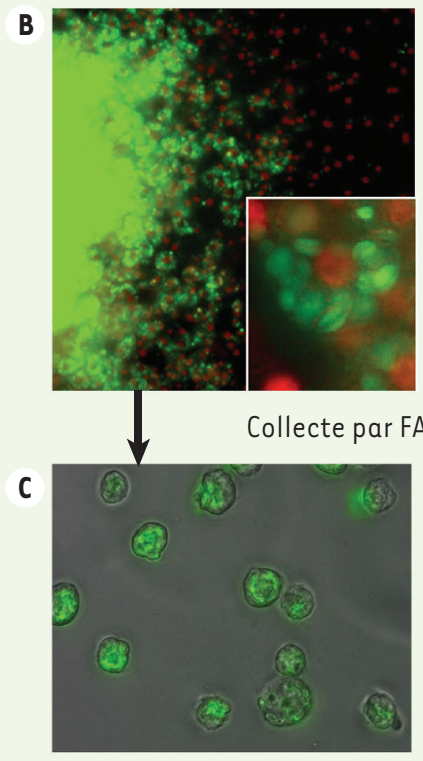

« nourries » avec des microbilles, et des cellules de types divers, correspondant aux cellules non fluorescentes, à partir d'éponges marquées par les deux traitements simultanés. Nous avons extrait instantanément les ARN messagers contenus dans ces fractions et les avons quantifiés par séquençage ARN. Nous avons ainsi identifié les gènes spécifiquement exprimés par les archéocytes. Nous avons ensuite cherché des gènes orthologues ${ }^{2}$ surexprimés dans les uPrisC d'autres lignées de métazoaires en utilisant les transcriptomes d'uPrisc publiés pour le cnidaire ${ }^{3}$ Hydra vulgaris [8] et la planaire 4 Schmidtea mediterranea [9]. Nous avons ainsi reconstruit un répertoire de 180 gènes dont l'expression est conservée dans les uPriSC de métazoaires.

\footnotetext{
2 Provenant d'un ancêtre commun.

${ }^{3}$ Les cnidaires existent sous deux formes : les formes fixées (corail, anémone de mer) et les formes libres et mobiles (méduses).

${ }^{4}$ Les planaires sont des vers plats aquatiques.
}

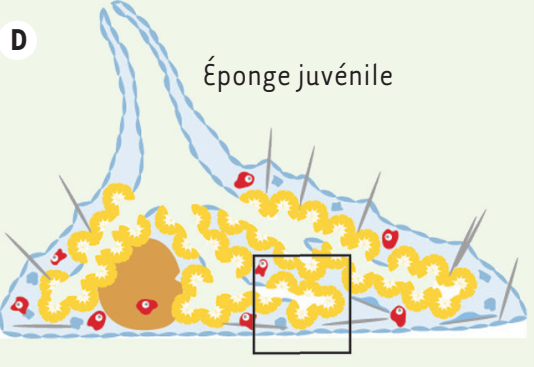

$$
\text { Choanocytes marqués (microbilles) }
$$$$
\varepsilon
$$
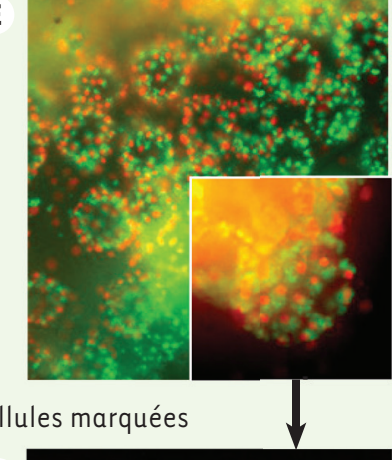

F

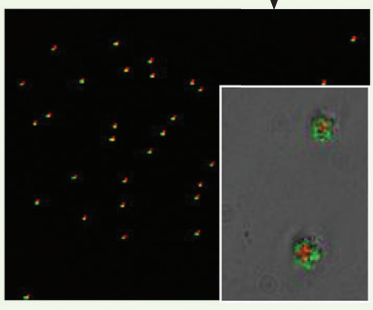

Comme nous l'attendions, ce répertoire contient une large proportion de gènes impliqués dans la réplication ou la réparation de I'ADN. De façon plus surprenante, il ne contient que trois facteurs de transcription, ce qui suggère que la grande majorité des facteurs de transcription associés aux cellules souches chez les animaux ne le sont pas depuis leur ancêtre commun. Par exemple, les facteurs de transcription ayant la capacité d'induire les cellules souches pluripotentes de mammifères - Sox2 (SRY [sex determining region $y$ ]-box 2), Nanog et 0ct4 (octamer-binding transcription factor 4) - n'ont pas d'orthologues en dehors des mammifères (pour Nanog et Sox2) ou des bilatériens ${ }^{5}$ (pour 0ct4), ce qui implique que leur rôle dans le maintien des cellules souches est une

${ }^{5}$ Les bilatériens ou artiozoaires sont une des subdivisions des eumétazoaires, contenant les protostomiens (arthropodes, mollusques, etc.) et les deutérostomiens (vertébrés, échinodermes).
Figure 1. Purification des archéocytes et des choanocytes de l'éponge Ephydatia fluviatilis. A. Schéma représentant une gemmule en cours de germination et les archéocytes (en rouge) migrant hors de la gemmule. $\boldsymbol{B}$. Région périphérique d'une éponge au stade représenté en $A$, dont les archéocytes ont été marqués par la rhodamine 123 (les plaquettes vitellines fluorescent en vert) et par du Hoechst (les noyaux fluorescent en rouge). C. Archéocytes récoltés après tri des cellules par FACS, toutes les cellules présentent de nombreuses plaquettes vitellines fluorescentes. D. Schéma représentant une éponge juvénile cinq jours après germination, la plupart des types cellulaires sont en place, y compris les nombreux choanocytes organisés en chambres choanocytaires (carré noir). $\varepsilon$. Plusieurs chambres choanocytaires montrant les choanocytes marquées par des microbilles fluorescentes (vertes) et du Hoechst (noyau marqué en rouge). $F$. Choanocytes récoltés après tri des cellules par FACS. FACS : fluorescence-activated cell sorting.

innovation des mammifères, et non une caractéristique ancestrale. Néanmoins, les archéocytes d'Ephydatia sur-expriment les facteurs de transcription GATA, p53, Glis (GLI-similar family zinc finger) et Myc, dont les orthologues ont des fonctions connues dans les cellules souches de mammifères. II est donc possible que les archéocytes d'éponges partagent avec les mammifères un réseau régulateur de l'expression des gènes, que l'on ne retrouve pas dans les uPriSC d'Hydra ou de Schmidtea. L'étude fonctionnelle de ces gènes chez les éponges est une voie très prometteuse pour comprendre les bases de la régulation génétique des cellules souches chez les animaux.

Contrairement aux facteurs de transcription, les régulateurs post-transcriptionnels sont très largement représentés dans le répertoire ancestral des uPrisC et comptent parmi les gènes les plus fortement exprimés. On y trouve par exemple des hélicases de la famille des DDX (DEAD-box proteins) qui régulent la traduction et divers aspects du métabolisme des ARN messager, ainsi que des gènes 


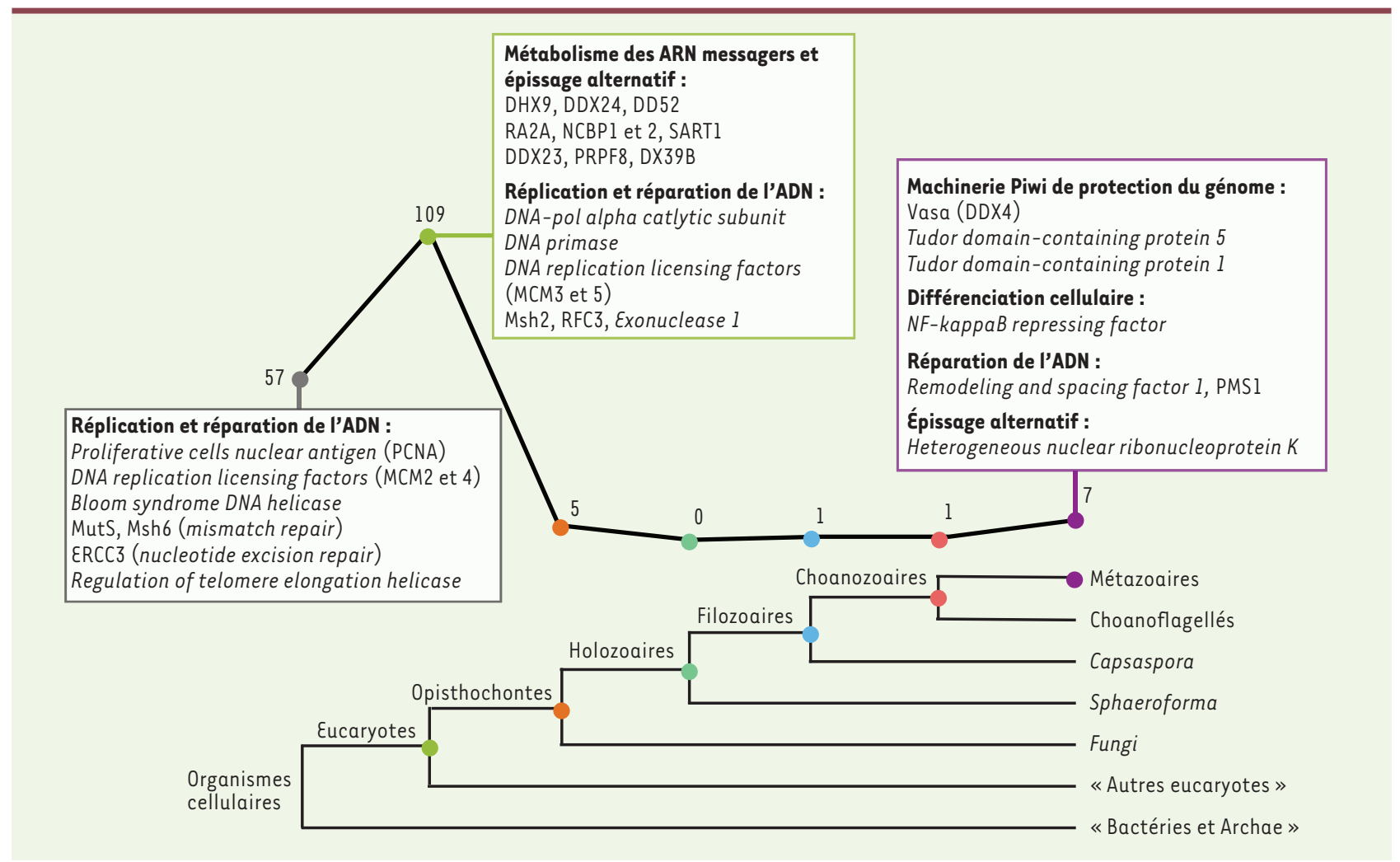

Figure 2. Profil phylogénétique des 180 gènes de la boîte à outils moléculaire ancestrale des cellules souches. En bas, un arbre phylogénétique du vivant montrant les nœuds auxquels les gènes ont été assignés. En haut, les chiffres représentent le nombre de gènes acquis à chaque nœud de l'arbre. Quelques gènes sont donnés en exemple.

codant des protéines à domaine RRM (RNA recognition motif), impliquées dans l'épissage alternatif. Le rôle important des régulateurs post-transcriptionnels commence à peine à être compris dans les cellules souches de mammifères [10] mais nos résultats tendent à prouver que ce mode de régulation génétique est ancestralement utilisé par les cellules souches. Il est probable que la régulation post-transcriptionnelle de l'expression des gènes représente un avantage pour les cellules souches car le maintien ou la sortie de l'état pluripotent, et l'engagement dans telle ou telle voie de différenciation cellulaire, sont des phénomènes qui requièrent une régulation précise et coordonnée des gènes exprimés par la cellule. En régulant leurs gènes au niveau de la traduction, les cellules souches sont capables de modifier plus rapidement le répertoire de protéines qu'elles expriment. Chez les mammifères, des régulateurs post-transcriptionnels sont regrou- pés au sein de régulons ${ }^{6}$ qui coordonnent le métabolisme des ARNm durant le cycle cellulaire et la réponse inflammatoire $[11,12]$. Dans le cas de la formation d'une éponge juvénile, une telle coordination est peut-être nécessaire pour que les archéocytes puissent construire en quelques jours une éponge fonctionnelle et organisée.

Pour comprendre comment la boîte à outils moléculaire ancestrale des celIules souches a été assemblée, nous avons retracé l'origine des 180 gènes la constituant, en cherchant les orthologues de ces gènes à travers l'arbre du vivant. II apparaît que les gènes régulant le cycle cellulaire sont d'origine très ancienne: on en trouve des orthologues jusque chez les bactéries et les archae. Les régulateurs post-transcriptionnels, quant à eux, sont pour la plupart hérités

${ }^{6}$ Ensemble fonctionnel de gènes dont l'expression est corégulée.

d'un ancêtre eucaryote. La majorité des composants de la boîte à outils ancestrale des cellules souches sont donc des gènes plus anciens que les cellules souches elles-mêmes, ayant été recrutés chez un ancêtre commun des animaux. Seuls 7 des 180 gènes ont été acquis en même temps que les cellules souches chez un ancêtre des animaux. Parmi ces 7 gènes, on trouve les gènes Vasa, $T d r d l$ (Tudor domain-containing 1) et Tdrd5 qui sont des composants d'une machinerie spéciale de protection du génome, la machinerie Piwi $[13,14](\rightarrow)$.

D’autres gènes spécifiques des métazoaires ont un rôle central dans cette

$(\rightarrow)$ Voir la Synthèse de S. Muller et al., $\mathrm{m} / \mathrm{s} \mathrm{n}^{\circ} 5$ mai 2013, page 487 machinerie et sont sur-exprimés dans les archéocytes, incluant Piwi lui-même, Tdrd9 et Tdrkh (Tudor and KH domain-containing). La machinerie Piwi est notamment impliquée dans la destruction d'éléments 
génétiques mobiles (les transposons) qui perturbent l'intégrité du génome. Or, protéger le génome est fondamental pour les uPrisc puisqu'elles produisent des cellules différenciées tout au long de la vie d'un organisme, mais également la lignée germinale par laquelle les gènes sont transmis aux générations suivantes. Notre travail montre donc que l'acquisition des cellules souches chez un ancêtre des animaux a été rendue possible non seulement par le recrutement de nombreux régulateurs post-transcriptionnels d'origine ancienne, mais aussi par l'acquisition d'une machinerie nouvelle de protection du génome, et que ces caractéristiques moléculaires ont accompagné l'évolution des cellules souches à travers I'histoire évolutive des animaux. $\diamond$ The ancestral gene repertoire of animal stem cells

\section{LIENS D’INTÉRÊT}

Les auteurs déclarent n'avoir aucun lien d'intérêt concernant les données publiées dans cet article.

\section{RÉFÉRENCES}

1. Tiozzo S, Copley R. Reconsidering regeneration in metazoans: an evo-devo approach. Front $\varepsilon$ col $\varepsilon v o l$ $2015 ; 3: 67$.

2. Philippe $H$, Derelle R, Lopez $P$, et al. Phylogenomics revives traditional views on deep animal relationships. Curr Biol 2009 ; 19 : 706-12

3. Pick KS, Philippe H, Schreiber F, et al. Improved phylogenomic taxon sampling noticeably affects nonbilaterian relationships. Mol Biol Evol 2010 ; 27 1983-7.

4. Fortunato SA, Adamski M, Adamska M. Comparative analyses of developmental transcription factor repertoires in sponges reveal unexpected complexity of the earliest animals. Mar Genomics $2015 ; 2$ : 121-9.

5. Alié A, Hayashi T, Sugimura I, et al. The ancestral gene repertoire of animal stem cells. Proc Natl Acad Sci USA 2015 ; 112 : ع7093-100.

6. Simpson TL. The cell biology of sponges. New York : Springer, 1984 : $662 \mathrm{p}$.

7. Solana J. Closing the circle of germline and stem cells: the primordial stem cell hypothesis. EvoDevo 2013 ; 4 2-16.
8. Hemmrich G, Khalturin K, Boehm AM, et al. Molecular signatures of the three stem cell lineages in hydra and the emergence of stem cell function at the base of multicellularity. Mol Biol Evol 2012 ; 29 : 3267-80.

9. Solana J, Kao D, Mihaylova Y, et al. Defining the molecular profile of planarian pluripotent stem cells using a combinatorial RNAseq, RNA interference and irradiation approach. Genome Biol 2012 ; 13 : R19.

10. Ye J, Blelloch R. Regulation of pluripotency by RNA binding proteins. Cell Stem Cell $2014 ; 15$ : 271-80.

11. Blackinton JG, Keene JD. Post-transcriptional RNA regulons affecting cell cycle and proliferation. Semin. Cell Dev Biol 2014 ; 34 : 44-54

12. Kafasla P, Skliris A, Kontoyiannis DL. Posttranscriptional coordination of immunological responses by RNA-binding proteins. Nat Immunol $2014 ; 15:$ 492-502.

13. Siomi MC, Mannen T, Siomi H. How does the royal family of Tudor rule the PIWI-interacting RNA pathway? Genes Dev 2010 ; 24 : 636-46.

14. Muller S, Raman Pandey R, Pillai RS. Les piARN forgent un système immunitaire pour le génome. Med Sci (Paris) 2013 ; $29:$ 487-94.

15. Damien Aubert D. Une nouvelle mégaclassification pragmatique du vivant. Med Sci (Paris) 2016; 32 : 497-9.

\section{NOUVELLE}

Le syndrome d'insensibilité
aux androgènes et activité
cérébrale chez la femme
Influence des hormones
et des chromosomes sexuels
Julie Bakker ${ }^{1}$, Judy van Hemmen ${ }^{2}$

> Dans l'espèce humaine, des différences entre sexes ont été observées à différents niveaux : les capacités cognitives [1], la structure du cerveau [2] et l'activité cérébrale associée à l'exécution de tâches cognitives ou émotionnelles [3]. Cependant, les mécanismes exacts impliqués dans la différenciation sexuelle du cerveau et du comportement chez l'homme sont encore fortement discutés. La théorie classique de la différenciation sexuelle du comportement est fondée sur l'étude de Phoenix et ses collègues [4]. Cette étude réalisée en 1959 chez le cobaye, montre que l'exposition aux androgènes, pendant certaines périodes spécifiques du développement, a des effets permanents (appelés organisateurs) en permettant l'expression de comportements sexuels typiquement masculins (comme le comportement de monte) en réponse aux stéroïdes sexuels à l'âge adulte. En revanche, l'absence d'androgènes, au cours de ces périodes, induira l'expression de comportements sexuels typiquement féminins (comme le comportement de lordose ${ }^{1}$ ). Des études ultérieures réalisées chez les rongeurs [5] $\rightarrow$ ont suggéré que les œstrogènes,

$(\rightarrow)$ Voir la Nouvelle de J. Bakker, $m / s n^{\circ} 5$, mai 2006, page 459

\footnotetext{
${ }^{1}$ La lordose est un réflexe moteur de courbure du dos chez la femelle, permettant de bien présenter la région génitale au mâle.
}

\author{
${ }^{1}$ Neuroendocrinologie, GIGA Neurosciences, \\ université de Liège, Avenue Hippocrate 15, \\ 4000 Liège, Belgique ; \\ ${ }^{2}$ Netherlands Institute for Neurosciences, \\ Meibergdreef 47, Amsterdam, Pays-Bas. \\ jbakker@ulg.ac.be
}

synthétisés dans le cerveau par l'enzyme aromatase, sont à l'origine d'un bon nombre de ces effets organisateurs des androgènes. Des études réalisées chez les primates [6], ainsi que des données obtenues chez l'homme concernant des troubles endocriniens rares [7], ont suggéré que, chez l'homme, les androgènes agissent directement sur le cerveau par leur liaison au récepteur aux androgènes. Dès d'adolescence, une fois la puberté installée, les taux circulants d'hormones sexuelles ont des effets « activateurs » sur le cerveau et le comportement et induisent de façon réversible les comportements qui ont été organisés au cours du développement. 